\title{
Design and Optimization of a Tiered Wireless Access Network
}

\author{
In Keun Son and Shiwen Mao \\ Dept. of Electrical and Computer Engineering, Auburn University, Auburn, AL \\ Email: soninkeun@auburn.edu, smao@ieee.org
}

\begin{abstract}
Although having high potential for broadband wireless access, wireless mesh networks are known to suffer from throughput and fairness problems, and are thus hard to scale to large size. To this end, hierarchical architectures provide a solution to this scalability problem. In this paper, we address the problem of design and optimization of a tiered wireless access network. At the lower tier, mesh routers are clustered based on traffic demands and delay requirements. The cluster heads are equipped with wireless optical transceivers and form the upper tier free space optical (FSO) network. We first present a plane sweeping and clustering algorithm aiming to minimize the number of clusters. PSC sweeps the network area and captures cluster members under delay and traffic load constraints. We then present an algebraic connectivity-based formulation for FSO network topology optimization and develop a greedy edgeappending algorithm that iteratively inserts edges to maximize algebraic connectivity. The proposed algorithms are analyzed and evaluated via simulations, and are shown to be highly effective as compared to the performance bounds derived in this paper.
\end{abstract}

\section{INTRODUCTION}

Wireless mesh networks (WMN) have emerged as a promising technology for providing ubiquitous broadband wireless access to mobile users, and for quick and easy extension of local area networks into a wide area [1]. As a result, recent years have witnessed significant growth in WMN research and deployment. However, WMNs are also known to suffer from the scalability problem. In [2], Jun and Sichitiu show that the per node throughput decreases as $O(1 / n)$, where $n$ is the number of nodes. In a recent work [3], the authors demonstrate that starvation occurs even in the simple scenario where one-hop flows contend with two-hop flows for gateway access. This is largely due to the inefficiency and bi-stability of existing MAC protocols, as well as high penalty for multi-hop flows to re-capture system resources [3].

To this end, hierarchical network architectures provide an effective solution to the scalability problem, as demonstrated in the Internet and wireless sensor networks [4]. Specifically, it is shown in [5] that by placing a sparse network of $m$ base stations (connected with high-bandwidth wired links) in an $n$-node ad hoc network, the asymptotic throughput capacity increases linearly with $m$ if $m$ grows faster than $\sqrt{n}$.

In this paper, we investigate the design and optimization of a tiered wireless access network, as illustrated in Fig. 1. The lower tier consists of a WMN with a large number of mesh routers providing wireless access to mobile users. To mitigate the scalability problem, we group mesh routers into clusters with bounded diameter. Traffic from/to the cluster members are aggregated and routed through the cluster head, which are equipped with free space optical (FSO) transceivers with multi-gigabit data rates and multi-kilometer ranges. With this architecture, resource contention mainly occurs within the cluster and end-to-end hop counts are greatly reduced due to the use of FSO links. Compared to the solution in [5], FSO links are also easier to deploy than wired links, and can be easily rearranged when traffic requirements change. The challenging task of QoS provisioning (e.g., delay and throughput) can be greatly simplified.

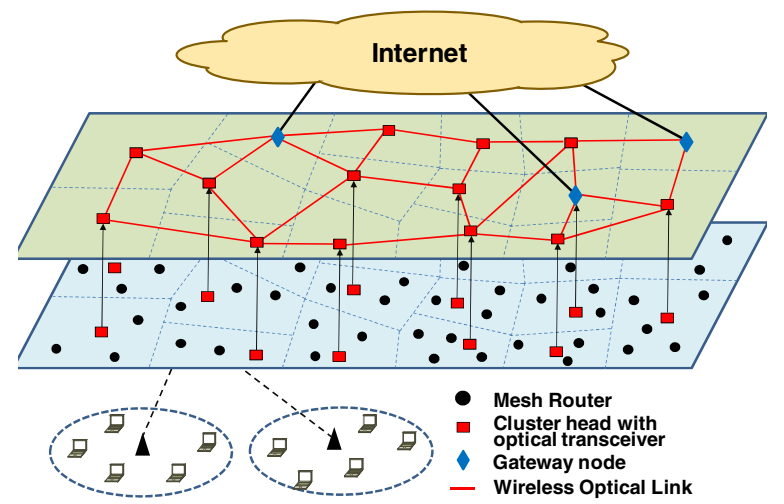

Fig. 1. Reference architecture for the tiered wireless access network.

FSO communication systems provide cost-effective, licensefree, and high-bandwidth links for the upper tier. FSO links require line-of-sight (LOS) and are point-to-point connections. They are immune to electromagnetic interference and are secure due to point-to-point connection with narrow beam divergence. However, FSO links are subject to impairments in the open-air transmission medium, such as path loss, atmospheric turbulence, obstacles, and beam misalignment. It is highly important to design topologies with rich connectivity for coping with such impairments.

We address the challenging problem of network planning for the tiered system. We take a divide-and-conquer approach to break it down into two sub-problems. The first sub-problem is cluster formation in the underlying WMN. The objective is to minimize the number of clusters (i.e., cost) while satisfying the delay and traffic load requirements. The second subproblem is how to design the topology of the upper tier FSO network to achieve maximum connectivity for a given 
number of edges. The two sub-problems are coupled with the common objectives of minimizing the cost and maximizing the reliability of the tiered system, while satisfying the QoS requirements. The formulated sub-problems belong to the class of NP-hard problems [6]. The large problem sizes also exclude sub-optimal solution procedures with high complexity [7], [8].

We take a graph theoretic approach to the problem of design and optimization of the tiered access network. First, we develop a plane sweeping and clustering (PSC) algorithm that sweeps the network area and captures cluster members one after another under delay and traffic load constraints. PSC chooses cluster members by manipulating the adjacency matrix and hop-count matrix of the underlying graph. Second, we present an algebraic connectivity-based formulation for FSO network topology optimization and develop a greedy edgeappending (GEA) algorithm that iteratively inserts edges to maximize algebraic connectivity. The proposed algorithms are analyzed with regard to complexity and performance bounds, and evaluated via simulations. They are shown to be highly effective for solving the network design and optimization problem as compared to the performance bounds developed in this paper.

The rest of this paper is organized as follows. We present the system model in Section II. The design and optimization problems are formulated in Section III. We present PSC and GEA, and analyze their performance in Sections IV and V, respectively. Simulation results are presented in Section VI and related work is discussed in Section VII. Section VIII concludes the paper.

\section{SYSTEM MODEL}

\section{A. Tiered Network Model}

As shown in Fig. 1, the lower tier of the wireless access network is a WMN consisting of $n$ mesh routers that provide access to mobile users [1]. The mesh routers use broadcast radio transceivers and each covers a small service area. We assume the mesh routers form a connected network. A small fraction of the mesh routers are gateway nodes with connection to the Internet. Traffic to/from the Internet is routed through the gateways, while traffic between a pair of mesh routers are relayed through multi-hop routes. The traffic demand is characterized by a given traffic matrix, denoted by $\mathbf{F}$, where element $[\mathbf{F}]_{i j}$ is the required load from mesh router $i$ to $j$.

WMNs are known to suffer from throughput and fairness problems [3]. They are hard to scale to large sizes, resulting in the so-called "a-few-hop" wireless networks. Although increasing the link rate helps, it is not practical to have FSO transceivers at each mesh router, due to the cost constraint and the mismatch on data rate and range between radio and FSO links. To this end, hierarchical structures provide an effective solution [5]. We assume the mesh routers are grouped into clusters, their traffic is aggregated at the cluster heads, and cluster heads are connected with high speed FSO links to provide fast lanes for the aggregate traffic.

The upper tier network is designated to cover wide range with high data rate. FSO provides license-free and high- bandwidth links for this purpose. We assume each cluster head is equipped with multiple FSO transceivers for rich connectivity, and the links are full duplex. The link capacity from cluster head $i$ to $j$ is denoted by $C_{i j}$. Due to LOS and focused transmissions, we assume $C_{i j}=C_{j i}$, for all $i \neq j$.

The main design considerations for FSO networks are cost and reliability. Available FSO systems cost from $\$ 10,000$ USD to $\$ 25,000$ USD for medium to long link ranges [9]. It is important to minimize the deployment cost of FSO networks. In addition, FSO links are also susceptible to atmospheric turbulence, unexpected tiny obstacles, and beam misalignment. Therefore, it is also important to achieve rich connectivity when designing the topology of the FSO network.

We assume three operation modes for the tiered access network: (i) Mode I: traffic is aggregated at cluster heads and forwarded through FSO links; (ii) Mode II: when an FSO link is temporarily blocked, a multipath routing protocol will redirect traffic through alternative paths; and (iii) Mode III: during severe weather conditions such as heavy snow, a large portion of the FSO links may be blocked. The lower tier WMN will backoff to the normal mesh networking mode with radio communications and multi-hop relays. Experimental results from three major U.K. cities show that atmospheric attenuation was constantly low over $99 \%$ of the time [10]. So the tiered network will work in Modes I and II most of the time.

\section{B. FSO Channel Model}

We assume that point-to-point wireless optical transceivers are used for the cluster heads in the outdoor field. Practical FSO communication systems usually adopt intensity modulation with direct detection (IM/DD) with On-Off Keying (OOK). There are several impairing factors through a LOS path, such as attenuation, atmospheric turbulence, unexpected tiny obstacles, and beam misalignment. In this paper, we consider atmospheric turbulence as characterized by the refractive-index structure parameter.

Several irradiance distributions have been used for modeling a light beam propagating through turbulent air. The log-normal distribution is shown to be highly accurate for weak weather turbulences [11]. The marginal distribution of received light intensity can be modeled as $f_{I}(I)=$ $\frac{1}{2 \sigma_{X} I} \frac{1}{\sqrt{2 \pi}} \exp \left\{-\frac{\left(\ln (I)-\ln \left(I_{0}\right)\right)^{2}}{8 \sigma_{X}^{2}}\right\}$, where $I_{0}$ is the average received intensity and $\sigma_{X}^{2}$ is the variance of the logamplitude fluctuation. The variance has the form $\sigma_{X}^{2}=$ $0.30545\left(\frac{2 \pi}{\lambda}\right)^{7 / 6} C_{n}^{2}(\eta) z^{11 / 6}$, where $\lambda$ is the wavelength, $C_{n}^{2}(\eta)$ is the index of refraction structure parameter with constant altitude $\eta$, and $z$ is the transmission distance.

The received optical intensity of fixed FSO links mainly depends on $C_{n}^{2}(\eta)$. For atmospheric channels near the ground, e.g., $\eta<18.5 \mathrm{~m}, C_{n}^{2}$ ranges from $10^{-13} \mathrm{~m}^{-2 / 3}$ to $10^{-17}$ $\mathrm{m}^{-2 / 3}$ for strong to weak atmospheric turbulences, with typical value $10^{-15} \mathrm{~m}^{-2 / 3}$ [11]. Under log-normal fading, the reliability of an FSO link can be computed as

$$
\Gamma_{i j}=\operatorname{Pr}\left\{I \geq I_{t h}\right\}=\frac{1}{2}-\frac{1}{2} \operatorname{erf}\left(\frac{\ln \left(I_{t h}\right)-\ln \left(I_{0}\right)}{2 \sigma_{X} \sqrt{2}}\right),
$$


where $I_{t h}$ is a threshold of received signal intensity. For fixed ratio of $\left(I_{t h} / I_{0}\right), \Gamma_{i j}$ is determined by the standard deviation $\sigma_{X}$, which is strongly influenced by weather conditions (i.e., a decreasing function of $C_{n}^{2}(\eta)$ ). With a suitable threshold $\Gamma_{t h}$, we define the weight of an FSO link as:

$$
\omega_{i j}= \begin{cases}\Gamma_{i j}, & \text { if } \Gamma_{i j} \geq \Gamma_{t h} \\ 0, & \text { otherwise. }\end{cases}
$$

Due to LOS and focused transmission, we assume that the links are symmetric, i.e., $\Gamma_{i j}=\Gamma_{j i}$ for all $i \neq j$. The main factors affecting link weights are the transmission distance and atmospheric turbulence. If the nominal capacity for all FSO links is $C_{o p t}$, the effective capacity of an FSO link is $C_{i j}=$ $\omega_{i j} \cdot C_{o p t}$ considering the transmission impairments. The upper tier FSO network, therefore, can be modeled as a undirected and weighted graph $G(V, E)$, where $V$ represents the set of cluster heads and $E$ represents the set of FSO links.

\section{Problem Statement}

In this section, we formulate the design and optimization problem for the tiered wireless access network. To make the problem tractable, we consider the following two subproblems: (i) the optimized clustering problem, on partitioning the underlying mesh network into clusters; and (ii) the topology optimization problem, on designing the upper tier FSO network topology. These two sub-problems are integrated with the overall objectives of minimizing the cost and maximizing the reliability of the tiered wireless network, while satisfying the traffic and delay requirements.

\section{A. Optimized Clustering Problem for the Lower Tier}

The first step is to partition the underlying wireless routers into clusters, while considering delay and traffic demand requirements. End-to-end delay consists of queueing delay, processing delay, and transmission (and retransmission) delay induced at each hop. For network planning purpose, we consider time averages of the delay components. This is because clustering is performed at relatively large timescales for which time averages matters. There is no need (or, it would be too costly) to adapt clusters in response to, say, queueing delay fluctuations at small timescales. As in prior work [12], we translate the delay requirement to a bound $h_{\max }$, i.e., the maximum hop count of the shortest path from any wireless router to its cluster head.

Assume the traffic matrix $\mathbf{F} \in \mathcal{R}^{n \times n}$ is given for the mesh network. When the clusters are formed, the aggregate incoming and outgoing traffic of cluster $M_{i}$, denoted by $f_{o, M_{i}}$ and $f_{M_{i}, o}$ respectively, can be computed as: $f_{o, M_{i}}=\sum_{j=1, j \notin M_{i}}^{n} \sum_{i \in M_{i}}[\mathbf{F}]_{j i}$ and $f_{M_{i}, o}=$ $\sum_{i \in M_{i}} \sum_{j=1, j \notin M_{i}}^{n}[\mathbf{F}]_{i j}$. Some operators may enforce a bound on traffic load, $f_{\max }$, for each cluster, i.e.,

$$
f_{M_{i}}=\max \left\{f_{o, M_{i}}, f_{M_{i}, o}\right\} \leq f_{\max } .
$$

Assuming that the transmission ranges of mesh radios are identical disks with radius $r$. For network design purpose, we focus on average link quality over relatively large timescales and ignore fast variations of channel quality. The mesh network can be represented by a graph $G$, with vertices representing mesh routers and edges representing radio links among neighboring mesh routers. The adjacency matrix $\mathbf{A} \in \mathcal{R}^{n \times n}$ of graph $G$ can be derived for given node locations and $r$. We have the following fact from graph theory.

Fact 1: Let $\mathbf{A}$ be the adjacency matrix of graph $G$. The number of walks from vertex $i$ to $j$ in $G$ with legnth $k$ is $\left[\mathbf{A}^{k}\right]_{i j}[13]$.

Recall that cluster diameters are bounded by $h_{\max }$. So we only concern with the maximum hop count $h_{\max }$, i.e., up to the $\left(h_{\max }\right)$-th power of $\mathbf{A}$. From Fact 1, the hop count of the shortest path between two nodes $i$ and $j$ can be computed as:

$$
h_{i j}= \begin{cases}\min \{k\}, & \text { if }\left[\mathbf{A}^{k}\right]_{i j}>0 \text { for } k \in\left[1, \cdots, h_{\text {max }}\right] \\ 0, & \text { otherwise. }\end{cases}
$$

We can define a hop-count matrix for graph $G$, denoted as $\mathbf{H} \in \mathcal{R}^{n \times n}$, with element $[\mathbf{H}]_{i j}=h_{i j}$, the hop count of the shortest path from node $i$ to $j$, for $i, j \in[1, \cdots, n]$.

Let binary variable $y_{i}$ be the indicator whether the $i$ th mesh router is chosen to be a cluster head and let $x_{i j}$ be the indicator whether mesh router $i$ is associated with cluster head $j$. The clustering problem can be formulated as a 0-1 integer linear programming (ILP) problem as:

$$
\text { minimize: } n_{c}=\sum_{i=1}^{n} y_{i}
$$

subject to:

$$
\begin{array}{ll}
\sum_{j=1}^{n} x_{i j}=1, & \text { for all } i \\
\sum_{j=1}^{n} x_{i j} \cdot h_{i j} \leq h_{\max }, & \text { for all } i \\
f_{M_{i}} \leq f_{\max }, & \text { for all } M_{i} \\
x_{i j} \in\{0,1\}, y_{i} \in\{0,1\}, & \text { for all } i, j .
\end{array}
$$

The main objective is to minimize the number of clusters to reduce the cost of the FSO network, under delay and traffic load constraints. Constraint (6) requires that each mesh router is assigned to one cluster. Constraint (7) indicates that the hop counts from all cluster members to the cluster head is bounded by $h_{\max }$. Constraint (8) represents the bound on the aggregated incoming and outgoing traffic for a cluster.

Once the clusters are formed, the new traffic matrix for the inter-cluster traffic, denoted by $\mathbf{F}^{\prime}$, can be derived as:

$$
\left[\mathbf{F}^{\prime}\right]_{i j}= \begin{cases}y_{i} y_{j} \sum_{a=1}^{n} \sum_{b=1}^{n} x_{a i} x_{b j}[\mathbf{F}]_{a b}, & \text { if } i \neq j \\ 0, & \text { otherwise. }\end{cases}
$$

The number of FSO transceivers at cluster head $i$, denoted as $K_{i}$, can be determined from $\mathbf{F}^{\prime}$, as:

$$
K_{i}=\max \left\{k_{\min }, \min \left\{k_{i}, k_{\max }\right\}\right\},
$$

where $k_{\min } \in\left[1, \cdots, k_{\max }\right]$ is a predefined lower bound on node degrees, $k_{i}=\left\lceil f_{M_{i}} /\left(\Gamma_{t h} \cdot C_{o p t}\right)\right\rceil$, and $k_{\max }=$ $\left\lceil f_{\max } /\left(\Gamma_{t h} \cdot C_{o p t}\right)\right\rceil$. In the following topology optimization problem, $K_{i}$ will be the degree for cluster head $i$. As will be shown in (12), the connectivity of a graph $G$ is upper bounded by its minimum degree $\delta(G)$. Here we enforce a minimum value $k_{\min }$ for $\delta(G)$ to avoid trivial cases with 
poor connectivity (e.g., stubs or trees). Once the degrees for the cluster heads are derived, we have the following facts from graph theory, which will be useful for the FSO network topology optimization problem.

Fact 2: The sum of node degrees is equal to twice of the total number of edges, i.e., $\sum_{i=1}^{n} K_{i}=2 \cdot|E|$ [13].

Fact 3: The number of edges of a simple graph (i.e., loopfree and without multiple edges) is upper bounded by $\left(\begin{array}{l}n \\ 2\end{array}\right)$ [13].

\section{B. Topology Optimization Problem for the Upper Tier}

The next step is to optimize the topology of the upper tier FSO backbone. As discussed, the FSO network can be modeled as a weighted simple graph $G(V, E)$ with $|V|=n_{c}$ vertices and $|E|=m$ edges. The problem is to find a simple graph with vertex set $V$ that achieves maximum connectivity for robustness to atmosphere turbulence and link failures, while satisfying node degree constraints (11).

There are several graph connectivity measures, such as $k$-vertex/edge connectivity and bisection connectivity [13]. We consider algebraic connectivity from spectral graph theory [14]. We first introduce algebraic connectivity and its properties, and then formulate the topology optimization problem.

1) Algebraic Connectivity Preliminaries: We adopt algebraic connectivity as a measure of network reliability, since it is amenable to mathematical modeling. The algebraic connectivity of a graph $G$ is defined to be the second smallest eigenvalue of the Laplacian matrix $\mathbf{L}$ of graph $G$, denoted as $\lambda_{2}(\mathbf{L})$, where $\mathbf{L}=\mathbf{L}(G)$ [14]. The value of $\lambda_{2}(\mathbf{L})$ is a nice indication of graph connectivity with the following facts.

Fact 4: A graph is connected if and only if $\lambda_{2}(\mathbf{L})>0$ [15].

Fact 5: The number of zero eigenvalues for $\mathbf{L}(G)$ is equal to the number of disjoint components of a graph $G$ [15].

Fact 6: For two graphs $G_{1}=\left(V, E_{1}\right)$ and $G_{2}=\left(V, E_{2}\right)$ with $E_{1} \subseteq E_{2}$, we have $\lambda_{2}\left(\mathbf{L}_{1}\left(G_{1}\right)\right) \leq \lambda_{2}\left(\mathbf{L}_{2}\left(G_{2}\right)\right)$ [15].

Algebraic connectivity is closely related to other connectivity measures. Consider a connected graph $G$ that is not complete. Let $K_{v}(G)$ denote the vertex connectivity and $K_{e}(G)$ denote the edge connectivity. The following inequality condition holds true [15]:

$$
\lambda_{2}\left(L_{2}\right) \leq K_{v}(G) \leq K_{e}(G) \leq \delta(G),
$$

where $\delta(G)$ is the minimum degree of the graph. Algebraic connectivity is also related to the number of vertices and graph diameter $D$ as $\lambda_{2}(G) \geq 4 /(D \cdot|V(G)|)$ [16]. Therefore, we can bound algebraic connectivity as

$$
\frac{4}{D \cdot|V(G)|} \leq \lambda_{2}(G) \leq \delta(G)
$$

Algebraic connectivity and its two bounds are all nondecreasing as more edges are added.

2) Topology Optimization Based on Algebraic Connectivity: As a result of the clustering problem, the $n_{c}$ cluster heads form an upper tier FSO network, which is modeled as a weighted graph $G(V, E)$. Each vertex $v_{i} \in V$ represents a cluster head with degree $K_{i}$, given in (11) as determined by the delay and traffic load requirements. The total number of edges is
$m=|E|=(1 / 2) \sum_{i=1}^{n_{c}} K_{i}$. Let the set of potential edges be $E_{\text {pot }}$ (i.e., set of edges with positive weight) and we have $E \subseteq$ $E_{\text {pot }}$. The topology design problem is to choose $E$ from $E_{\text {pot }}$ such that (i) the degree of each vertex is satisfied and (ii) the algebraic connectivity is maximized. Since the transmission range of FSO links is usually much longer than the distance between adjacent cluster heads, we expect $\left|E_{\text {pot }}\right| \gg \sum_{i=1}^{n_{c}} K_{i}$.

We follow the Laplacian matrix definition in [17]. Assume edge $l$ connects two distinct vertices $v_{i}$ and $v_{j}, l \in[1, \cdots, m]$, $v_{i}, v_{j} \in V$. We also denote edge $l$ as $(i, j)$ when there is need to distinguish the endpoints. We define $\mathbf{a}_{l} \in \mathcal{R}^{n_{c}}$ of the unweighted incidence matrix $\Lambda \in \mathcal{R}^{n_{c} \times m}$ of graph $G$ as

$$
\left[\mathbf{a}_{l}\right]_{k}= \begin{cases}+1, & \text { if } k=i \text { and } \omega_{i j}>0 \\ -1, & \text { if } k=j \text { and } \omega_{i j}>0 \\ 0, & \text { otherwise. }\end{cases}
$$

The $n_{c} \times n_{c}$ weighted Laplacian matrix $\mathbf{L}$ of graph $G$ is: $\mathbf{L}(G)=\sum_{l=1}^{m} \omega_{l} \cdot \mathbf{a}_{l} \cdot \mathbf{a}_{l}^{T}=\boldsymbol{\Lambda} \cdot \operatorname{diag}(\omega) \cdot \boldsymbol{\Lambda}^{T}$, where $\operatorname{diag}(\omega) \in \mathcal{R}^{m \times m}$ is a diagonal matrix with diagonal elements $\omega_{l}, l=1, \cdots, m$. It follows from the definition that $L(G)$ is a positive semi-definite matrix, i.e., $\mathbf{L}(G) \succeq 0$. Its smallest eigenvalue, $\lambda_{1}(\mathbf{L})$, is zero with eigenvector $\mathbf{1}=[1,1, \cdots, 1]^{T}$. The eigenvalues also satisfy the following inequality condition: $0=\lambda_{1} \leq \lambda_{2} \leq \lambda_{3} \leq \cdots \leq \lambda_{n_{c}}$. Since we consider connected graphs, it follows from Facts 4 and 5 that there is only one zero eigenvalue. Therefore we have

$$
0=\lambda_{1}<\lambda_{2} \leq \lambda_{3} \leq \cdots \leq \lambda_{n_{c}},
$$

where $\lambda_{2}(\mathbf{L})$ is the algebraic connectivity of graph $G$.

The topology optimization problem for the upper tier FSO network can be formulated as follows. ${ }^{1}$

$$
\begin{aligned}
\text { maximize: } & \lambda_{2}(\mathbf{L}) \\
\text { subject to: } & m=\frac{1}{2} \sum_{i=1}^{n_{c}} K_{i} \leq\left(\begin{array}{c}
n_{c} \\
2
\end{array}\right) \\
d_{i} & =K_{i}, \text { for all } i \\
& E \subseteq E_{\text {pot }},
\end{aligned}
$$

where $d_{i}$ is the non-weighted degree of node $i$. To optimize the FSO network topology, we populate edge set $E$ by selecting edges from $E_{\text {pot }}$, such that the algebraic connectivity of the resulting graph $G$ is maximized and the delay and traffic load requirements are satisfied. The first constraint is from Facts 2 and 3. The second constraint is on the degree of each cluster head, such that the incoming and outgoing traffic from that cluster can be served.

When the problem is solved, each potential link $l \in E_{\text {pot }}$ will either be chosen to be included in $E$ or not chosen. Define binary variables $x_{l}$ as:

$$
x_{l}= \begin{cases}1, & \text { if potential edge } l \text { is included in } E \\ 0, & \text { otherwise. }\end{cases}
$$

\footnotetext{
${ }^{1}$ It is worth noting that $K_{i}$ is determined using $\Gamma_{t h}$ as in (11), rather than the exact link reliability $\Gamma_{i j} \geq \Gamma_{t h}$. As a result, some redundancy is provided for the capacity of cluster $i$, which is useful to accommodate fluctuations in the instantaneous cluster $i$ load. If such redundancy is unnecessary, we can modify the formulation by replacing constraint (18) with $C_{o p t} \cdot \sum_{j:(i, j) \in E} \omega_{i j} \geq$ $\min \left\{f_{\max }, f_{M_{i}}\right\}$, for all $i$.
} 
Then $E_{\text {pot }}$ can be translated into a boolean vector $\mathbf{x} \in$ $\{1,0\}^{m_{\text {pot }}}$, where $m_{\text {pot }}$ is the cardinality of $E_{\text {pot }}$. The topology optimization problem can be reformulated into a 0-1 ILP problem as:

$$
\begin{array}{ll}
\operatorname{maximize}: & \lambda_{2}\left(\sum_{l=1}^{m_{p o t}} x_{l} \cdot w_{l} \cdot \mathbf{a}_{l} \cdot \mathbf{a}_{l}^{T}\right) \\
\text { subject to: } & \mathbf{1}^{T} \cdot \mathbf{x}=\frac{1}{2} \sum_{i=1}^{n} K_{i} \leq\left(\begin{array}{c}
n_{c} \\
2
\end{array}\right) \\
& d_{i}=K_{i}, \text { for all } i \\
& \mathbf{x} \in\{0,1\}^{m_{\text {pot }}}, \omega_{l}=\omega_{i j}, l \sim(i, j) .
\end{array}
$$

The solution to the problem is a boolean vector $\mathrm{x}$ that maximizes the algebraic connectivity of the resulting graph.

\section{Remarks}

Both formulated problems belong to the class of 0-1 ILP. Such problems are classified as NP-hard, and in fact the decision version was one of Karp's 21 NP-complete problems [6]. Since exhaustive search can only apply to small-scale problems, approximation and heuristic algorithms are needed for sub-optimal solutions. In [7], [8], the relaxed problem is solved using semidefinite program (SDP), which can handle moderate-sized problems (e.g., 1000 edges). Low complexity greedy perturbation heuristic algorithms and bounds are developed in [8] to overcome the complexity limit of SDP.

In the next two sections, we develop bounds and heuristic algorithms for the formulated problems. Specifically, we develop a plane sweeping and clustering (PSC) algorithm for the clustering problem and a greedy edge-appending (GEA) algorithm for the topology optimization problem, along with performance bounds. Both algorithms are evaluated via simulations in Section VI.

\section{ALGORITHM AND BOUND FOR OPTIMIZED Clustering}

The PSC algorithm is to continually sweep the network area to capture cluster members while satisfying the delay and load constraints, until every mesh router is assigned to a cluster. We then choose cluster head for each cluster formed. The objective is to minimize the number of clusters, thus reducing the deployment cost of the upper tier FSO network.

\section{A. Plane Sweeping and Clustering Algorithm}

The pseudo-code of the PSC algorithm is presented in Table I. Starting from the lower left corner of the network region, PSC iteratively sweeps the network area and forms a new cluster $M_{t}$ after each iteration $t$. Specifically, each iteration consists of three steps as follows.

1) Step 1: PSC first chooses a base node as starting point for the next cluster to be formed. It then explores the neighbors of the base node within $h_{\max }$ hops, and adds the neighboring nodes into the cluster, until one of the conditions, $D_{M_{t}} \leq$ $h_{\max }$ or $f_{M_{t}} \leq f_{\max }$, is violated (Lines 5-19 in Table I). $D_{M_{t}}$ is the cluster diameter of $M_{t}$ and can be computed as:

$$
D_{M_{t}}=\max _{i, j \in M_{t}, i \neq j}\left[\mathbf{H}_{t}\right]_{i j},
$$

TABLE I

The Plane Sweeping and Clustering (PSC) Algorithm

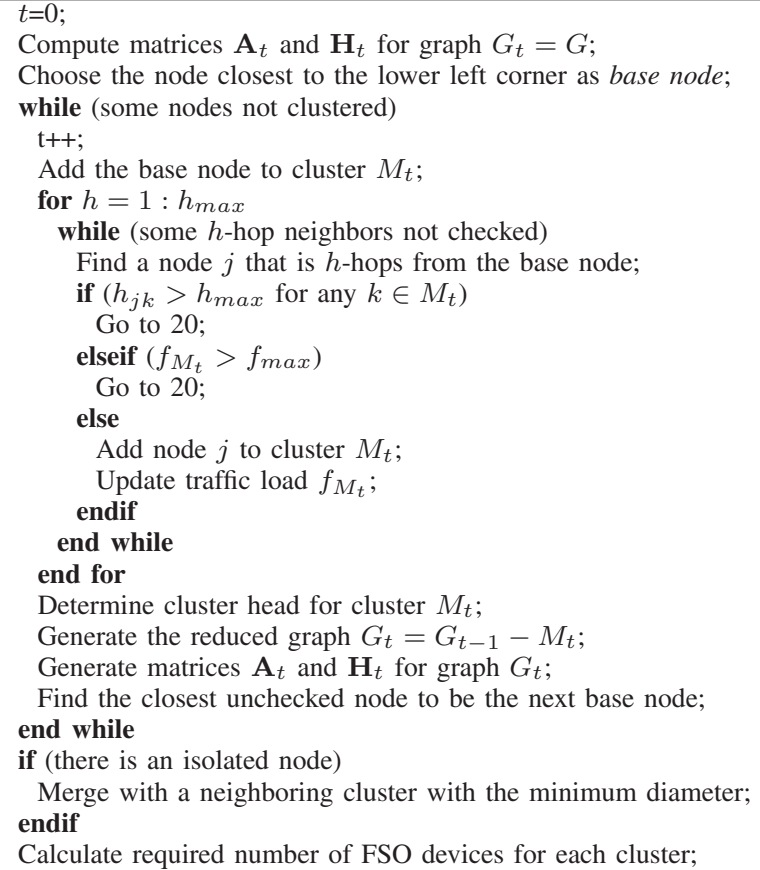

where $\mathbf{H}$ is the hop-count matrix for cluster $M_{t}$ as defined in Section III-A. Recall that $f_{M_{t}}=\max \left\{f_{o, M_{t}}, f_{M_{t}, o}\right\}$ is the larger one of the aggregate incoming or outgoing traffic load of cluster $M_{t}$, as given in (3).

It is worth noting that in the optimized clustering problem (5)-(9), the delay constraint is translated to $\sum_{j=1}^{n} x_{i j} \cdot h_{i j} \leq$ $h_{\max }$ for all $i \in V$. That is, the hop count of the shortest path from any cluster member to its cluster head should be upper bounded by $h_{\max }$. Since the cluster head could be located anywhere in the cluster (not necessarily at the center, see the discussion of Step 2), this condition is satisfied by bounding cluster diameter with $h_{\max }$ in PSC, such that the maximum hop count for any node pair in the cluster does not exceed $h_{\max }$. This also provides redundancy for accommodating fluctuations in the delay components.

2) Step 2: When a new cluster $M_{t}$ is formed, PSC will select a cluster head from nodes in $M_{t}$ (Line 20 in Table I). This node will be equipped with FSO transceivers and become a node in the upper tier FSO network.

Since all the incoming and outgoing traffic for a cluster will go through the cluster head, it should be judiciously chosen such that the relay traffic load within the cluster is minimized. If there is a gateway node in the cluster, it should be the cluster head, since all the traffic will finally be directed to and from there. If there are more than one gateway nodes in the cluster, we will split the cluster into multiple smaller clusters, each using a gateway node as cluster head. Each non-gateway node in $M_{t}$ will be associated with the closest gateway node in $M_{t}$. It is worth noting that this case rarely happens since the number of gateway nodes is usually small. If there is no gateway node in $M_{t}$, we select a node as cluster head by 
solving the following optimization problem:

$$
\underset{q \in M_{t}}{\arg \min }\left\{\sum_{i \in M_{t}, i \neq q} h_{i q}\left(\sum_{j \notin M_{t}, j=1}^{n}\left([\mathbf{F}]_{i j}+[\mathbf{F}]_{j i}\right)\right)\right\},
$$

where the overall relay load for the cluster's incoming and outgoing traffic is minimized.

3) Step 3: If there is still node not clustered, PSC will obtain a reduced graph $G_{t}$ by deleting the nodes in $M_{t}$ from graph $G_{t-1}$, and update the adjacency matrix $\mathbf{A}_{t}$ and hopcount matrix $\mathbf{H}_{t}$ for the reduced graph $G_{t}$. PSC then chooses in $G_{t}$ the closest node from the current base node point, as well as close to certain trajectory (horizontal or vertical), as the next base node, and will repeat Steps 1) and 2) to generate a new cluster (Line 21-23 in Table I).

\section{B. Lower Bound}

We next derive a lower bound on the objective value achieved by PSC. To avoid trivial cases, ${ }^{2}$ and without loss of generality, we assume that every point in the deployment area, $S$, is covered by at least one mesh router. The lower bound on the number of clusters is given in the following theorem. It will be useful for evaluating the performance of heuristic clustering algorithms, especially for large-scale networks.

Theorem 1: $n_{0}=\left\lceil 4 S /\left(\pi r^{2} h_{\max }^{2}\right)\right\rceil \leq n_{c}$ is a lower bound on the total number of clusters.

Proof: In order to minimize the number of clusters, the size of each cluster should be maximized. For given $h_{\max }$ and $f_{\max }$, there are two cases when a cluster is formed, i.e., the cluster size is confined by either (i) the maximum cluster diameter or (ii) the maximum aggregate traffic load.

(Case i) Since the cluster size is confined by $h_{\max }$, the minimum number of clusters can be obtained by forming each cluster with diameter $h_{\max }$. With transmission range $r$, the maximum Euclidean distance between any two cluster members is thus $r h_{\max }$. The maximum coverage of a cluster is $\pi r^{2} h_{\max }^{2} / 4$, which is a disk with radius $r h_{\max } / 2$. To cover the deployment area, the minimum number of clusters can be estimated as $\left\lceil 4 S /\left(\pi r^{2} h_{\max }^{2}\right)\right\rceil$.

(Case ii) Since the cluster size is confined by $f_{\max }$, the maximum cluster diameter bound is not violated when the cluster is formed. As a result, the Euclidean distance of the cluster diameter cannot be larger than $r h_{\max }$. Therefore, the minimum number of clusters for this case cannot be lower than that in Case (i).

Therefore, we conclude that the minimum number of clusters is $n_{0}=\left\lceil 4 S /\left(\pi r^{2} h_{\max }^{2}\right)\right\rceil$.

\section{Complexity Reduction}

PSC involves manipulation of several matrices. It can be shown to have a complexity of $O\left(n_{c} \cdot n^{2.376}\right)$, which mainly stems from matrix multiplications. In this section, we discuss techniques on further reducing the computational complexity.

\footnotetext{
${ }^{2}$ Consider the following trivial case: the lower bound is one if all the wireless routers are installed at the same location with low traffic load.
}

Theorem 2: Consider graph $G^{\prime}=G-k$ for $k \in V(G)$. For any $i \in V(G)$ such that $h_{i k} \geq h_{\max }$, we have $[\mathbf{H}]_{i j}=\left[\mathbf{H}^{\prime}\right]_{i j}$ for all $j \in V(G)$ and $j \neq k$.

Proof: For $i, k \in V(G)$ and $i \neq k$, assume that there is an $i k$-walk with minimum number of random walk $n$. If $h_{i k}>h_{\max }$, we have $h_{i k}=0$. Then, the $i k$-walk is denoted by $v_{i}=v_{0} e_{0} v_{1} e_{1} \cdots v_{n-1} e_{n-1} v_{n}=v_{k}$ for $n \leq h_{\max }$. For the reduced graph $G^{\prime}=G-k$, assume there is a vertex $j \neq k$. We consider the following three cases.

(Case i) Assume that $h_{i k}>h_{\max }$. If $h_{i j} \leq h_{\max }, h_{i j}^{\prime}$ is equal to $h_{i j}$ since the $i j$-walk does not include $v_{k}$ by the case condition $h_{i k}>h_{\max }$. If $h_{i j}>h_{\max }, h_{i j}^{\prime}$ is also equal to $h_{i j}$ (i.e., both are 0 ), since deleting a vertex does not reduce the number of any walk. Thus we have $[\mathbf{H}]_{i j}=\left[\mathbf{H}^{\prime}\right]_{i j}$.

(Case ii) Assume that $h_{i k}=h_{\max }$. If $h_{i j}<h_{\max }$, $h_{i j}^{\prime}$ is equal to $h_{i j}$ since the $i j$-walk does not include $v_{k}$ by the assumption $h_{i k}=h_{\max }$. If $h_{i j}=h_{\max }$, there should be another walk which does not go through $v_{k}$. If not, the condition, $h_{i j}=h_{\max }$, will be violated. For the condition $h_{i j}>h_{\max }, h_{i j}^{\prime}$ is also equal to $h_{i j}$ since both of them are 0 in this case. Thus $[\mathbf{H}]_{i j}=\left[\mathbf{H}^{\prime}\right]_{i j}$ holds true in this case.

(Case iii) Assume that $h_{i k}<h_{\max }$. If $h_{j k}<h_{\max }$ and $h_{i j}=h_{i k}+h_{k j}$, it is possible that the shortest $i j$-walk is unique and $v_{k}$ is in the path. It is possible to have $h_{i j}^{\prime} \neq h_{i j}$.

We conclude that $[\mathbf{H}]_{i j}=\left[\mathbf{H}^{\prime}\right]_{i j}$ when $h_{i k} \geq h_{\max }$.

Corollary 2.1: Consider graph $G^{\prime}=G-k$ for $k \in V(G)$. Assuming disjoint vertices $i, j, k \in V(G)$, it is possible to have that $h_{i j}^{\prime} \neq h_{i j}$, if $h_{i j}=h_{i k}+h_{j k}$ for $h_{i k}<h_{\max }$ and $h_{j k}<h_{\max }$.

Corollary 2.2: Consider graph $G^{\prime}=G-k$ for $k \in V(G)$. Assume that vertex $i, j, k \in V(G)$ are disjoint. Then $\left[\mathbf{H}^{\prime}\right]_{i j}$ can be obtained by the power calculation of an adjacency matrix, denoted as $\mathbf{A}^{\prime \prime}$, consisting of rows and columns corresponding to vertices $s$ and $t \in V(G)$, satisfying $h_{k s} \leq 2\left(h_{\max }-1\right)$ and $h_{k t} \leq 2\left(h_{\max }-1\right)$.

The proofs of the corollaries are omitted due to lack of space. Please refer to the journal version for details.

\section{BOUnd AND Algorithm FOR TOPOLOGY OPTIMIZATION}

The objective of topology design is to maximize the algebraic connectivity for the upper tier FSO network, while satisfying the traffic load requirements. We first derive a theoretic upper bound for algebraic connectivity, and then present the GEA algorithm, which is shown to achieve algebraic connectivity very close to the upper bound in Section VI.

\section{A. Theoretic Upper Bound}

In this section, we present an upper bound on the increase of algebraic connectivity achieved by appending an extra edge to a graph. This upper bound is useful for evaluating the performance of GEA, since the global optimal lies in between the GEA solution and the upper bound. Let $G(V, E)$ be a connected and weighted graph and let $G^{\prime}$ be a graph by adding an edge $l \sim(i, j)$ to $G$, denoted as $G^{\prime}=G+l$. The upper bound is given in the following. 
TABLE II

The Greedy Edge-Appending (GEA) Algorithm

\begin{tabular}{|c|c|}
\hline & Build a degree bounded minimum spanning tree; \\
\hline & $\begin{array}{l}\text { while (there remains an edge to be inserted) } \\
\text { Append the edge with the largest } \omega_{i j} \cdot\left(\nu_{i}-\nu_{j}\right)^{2} \text { among }\end{array}$ \\
\hline & $\begin{array}{l}\text { remaining edges in } E_{\text {pot }} ; \\
\text { - Conditioned on: }\left(d_{i}<K_{i}\right) \text { and }\left(d_{j}<K_{j}\right) \text {; } \\
\text { - Break a tie by considering the node pair with the minimum } \\
\text { degree first and then that with the maximum distance; }\end{array}$ \\
\hline & $\begin{array}{l}\text { Update the graph and the corresponding Laplacian matrix; } \\
\text { end while }\end{array}$ \\
\hline
\end{tabular}

Theorem 3: An upper bound on the algebraic connectivity of graph $G^{\prime}$ is given as:

$$
\lambda_{2}\left(G^{\prime}\right) \leq \min \left\{\lambda_{3}(G), \lambda_{2}(G)+\omega_{i j} \cdot\left(\nu_{i}-\nu_{j}\right)^{2}\right\},
$$

where $\nu_{i}$ and $\nu_{j}$ are the $i$ th and $j$ th elements of the normalized eigenvector $\mathbf{v}$ of $\mathbf{L}(G)$ corresponding to eigenvalue $\lambda_{2}(G)$.

Proof: Let $\mathbf{L}$ and $\mathbf{L}^{\prime}$ be the Laplacian matrix of $G$ and $G^{\prime}$, respectively. We first show that $\lambda_{2}\left(G^{\prime}\right) \leq \lambda_{3}(G)$. Assuming that graph $G$ has one component, we have $\mathbf{L}^{\prime}=\mathbf{L}+\omega_{l}$. $\mathbf{a}_{l} \cdot \mathbf{a}_{l}^{T}$. Since the Laplacian matrix $\mathbf{L}$ is symmetric positive semidefinite, it satisfy the following conditions: (i) $\mathbf{L}=\mathbf{L}^{T}$, (ii) $\mathbf{L} \succeq 0$, and (iii) $\mathbf{L} \cdot \mathbf{1}=\mathbf{0}$, where $\mathbf{0}=[0,0, \cdots, 0]^{T}$.

Let $\lambda_{1} \leq \lambda_{2} \leq \cdots \lambda_{n_{c}}$ be the eigenvalues of $\mathbf{L}$ and $\lambda_{1}^{\prime} \leq$ $\lambda_{2}^{\prime} \leq \cdots \lambda_{n_{c}}^{\prime}$ be the eigenvalues of $\mathbf{L}^{\prime}$. According to Theorem 3.2 in [16], we have $\lambda_{1} \leq \lambda_{1}^{\prime} \leq \lambda_{2} \leq \lambda_{2}^{\prime} \leq \lambda_{3} \leq \lambda_{3}^{\prime} \cdots \leq$ $\lambda_{n_{c}} \leq \lambda_{n_{c}}^{\prime}$. Therefore, it follows that $\lambda_{2}\left(G^{\prime}\right) \leq \lambda_{3}(G)$.

We next prove the second part. Let $\mathbf{v}=\left(\nu_{1}, \nu_{2}, \cdots, \nu_{n_{c}}\right)$ be the normalized eigenvector of $\mathbf{L}$ corresponding to $\lambda_{2}(\mathbf{L})$. According to the Courant-Fischer formula, the second smallest eigenvalue of $\mathbf{L}^{\prime}$ can be written as $\lambda_{2}\left(\mathbf{L}^{\prime}\right)=$ $\min _{\mathbf{x} \perp \mathbf{1}, \mathbf{x} \neq \mathbf{0}} \frac{\left\langle\mathbf{L}^{\prime} \cdot \mathbf{x}, \mathbf{x}\right\rangle}{\langle\mathbf{x}, \mathbf{x}\rangle}$, where $\langle\cdot, \cdot\rangle$ is the inner product of two vectors [16]. It follows that

$$
\begin{aligned}
\lambda_{2}\left(\mathbf{L}^{\prime}\right) & =\min _{\mathbf{x} \perp \mathbf{1}, \mathbf{x} \neq 0} \frac{\left\langle\mathbf{L}^{\prime} \cdot \mathbf{x}, \mathbf{x}\right\rangle}{\langle\mathbf{x}, \mathbf{x}\rangle} \leq \frac{\left\langle\mathbf{L}^{\prime} \cdot \mathbf{v}, \mathbf{v}\right\rangle}{\langle\mathbf{v}, \mathbf{v}\rangle} \\
& =\frac{\sum_{(a, b) \in E\left(G^{\prime}\right)} \omega_{a b} \cdot\left(\nu_{a}-\nu_{b}\right)^{2}}{\sum_{a \in V(G)} \nu_{a}^{2}} \\
& =\sum_{\{(a, b) \in E(G)\} \cup\{(i, j) \notin E(G)\}} \omega_{a b} \cdot\left(\nu_{a}-\nu_{b}\right)^{2} \\
& =\sum_{(a, b) \in E(G)} \omega_{a b} \cdot\left(\nu_{a}-\nu_{b}\right)^{2}+\omega_{i j} \cdot\left(\nu_{i}-\nu_{j}\right)^{2} \\
& =\lambda_{2}(\mathbf{L})+\omega_{i j} \cdot\left(\nu_{i}-\nu_{j}\right)^{2} .
\end{aligned}
$$

The third equality is due to the fact that $\mathbf{v}$ is a normalized vector. We have $\lambda_{2}\left(\mathbf{L}^{\prime}\right) \leq \lambda_{2}(\mathbf{L})+\omega_{i j}\left(\nu_{i}-\nu_{j}\right)^{2}$. Since both cases should be satisfied, we have that $\lambda_{2}\left(G^{\prime}\right) \leq$ $\min \left\{\lambda_{3}(G), \lambda_{2}(G)+\omega_{i j} \cdot\left(\nu_{i}-\nu_{j}\right)^{2}\right\}$.

\section{B. Greedy Edge-Appending Algorithm}

As discussed, the SDP approach has very high complexity and is only suitable for small and moderate problem sizes. It also requires a rounding heuristic to obtain a feasible solution, which may have an unpredictable performance [7], [8]. These make low complexity heuristics highly appealing.
We present a greedy algorithm GEA that iteratively inserts edges into the current graph. The pseudo-code is given in Table II. The procedure starts with $n_{c}$ vertices and a null edge set. Therefore, the initial value of $\lambda_{2}(\mathbf{L})$ is zero and will remain zero until the graph becomes connected (see Facts 4 and 5). To speed up the initialization phase, we first apply an enhanced version of Prim's algorithm to build a degree bounded minimum spanning tree [18]. Next, GEA iteratively picks an edge from the remaining edges in $E_{p o t}$ and appends it to the graph such that the algebraic connectivity is progressively improved.

Recall that $\mathbf{v}=\left(\nu_{1}, \nu_{2}, \cdots, \nu_{n_{c}}\right)$ is the normalized eigenvector corresponding to $\lambda_{2}(\mathbf{L})$. During each iteration, GEA chooses an edge with the largest $\omega_{i j} \cdot\left(\nu_{i}-\nu_{j}\right)^{2}$ value among all the remaining edges in $E_{\text {pot }}$. As shown in the proof of Theorem 3, $\omega_{i j} \cdot\left(\nu_{i}-\nu_{j}\right)^{2}$ is an upper bound on the algebraic connectivity increase achieved by adding edge $(i, j)$. This strategy will achieve the largest expected increase in $\lambda_{2}(\mathbf{L})$.

In the case of a tie where multiple edges have the same maximum $\omega_{i j} \cdot\left(\nu_{i}-\nu_{j}\right)^{2}$ value, GEA chooses the edge whose endpoint has the minimum degree. If there is a tie again, GEA chooses the edge with the largest distance. This strategy is motivated by the inequality condition, $\frac{4}{D \cdot n_{c}} \leq \lambda_{2}(G) \leq \delta(G)$, which implies that algebraic connectivity may be improved by increasing the minimum degree and/or by decreasing the diameter of the graph.

It takes $O\left(\left(n_{c}-1\right)^{2}\right)$ to build the minimum spanning tree with $n_{c}-1$ edges [18]. The complexity of appending the remaining $\left(m-n_{c}+1\right)$ edges mainly comes from computing eigenvector $\mathbf{v}$, each with complexity takes $O\left(n_{c}\right)$, for the remaining edges in $E_{\text {pot }}$. The overall GEA complexity is $O\left(\left(n_{c}-1\right)^{2}+\left(m-n_{c}+1\right) n_{c} m_{p o t}\right)$.

\section{Simulation Results}

In this section, we evaluate the performance of PSC and GEA. The algorithms are implemented in Matlab. We use fixed transmission range $r$ for the lower tier radio network, and simulate log-normal fading for the upper tier FSO links. During each simulation, mesh routers are uniformly distributed in a square region with randomly chosen gateways.

The traffic matrix is randomly generated as follows. Each mesh router generates two types of traffic: (i) Internet traffic that is forwarded to a gateway, and (ii) inter-cluster traffic that is delivered within the WMN. The inter-cluster traffic is randomly distributed among mesh routers and the Internet traffic is directed to the closest gateway. We assume the incoming Internet traffic rate is twice that of the outgoing Internet traffic (i.e., more downloading than uploading).

\section{A. Optimized Clustering Results}

We first evaluate the PSC performance with regard to number of clusters and computation reduction. We follow the setting in [12] to consider a small network with 175 mesh routers uniformly deployed in a $1,000 \times 1,000 \mathrm{~m}^{2}$ region. There are two gateway nodes. The radio transmission range is $r=100 \mathrm{~m}$, and the minimum distance between any two mesh 


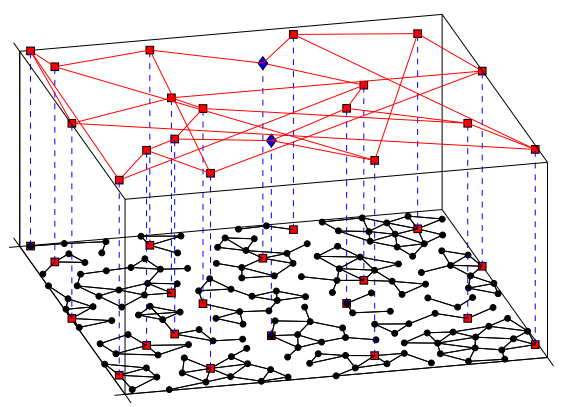

Fig. 2. A tiered network designed by PSC/GEA.

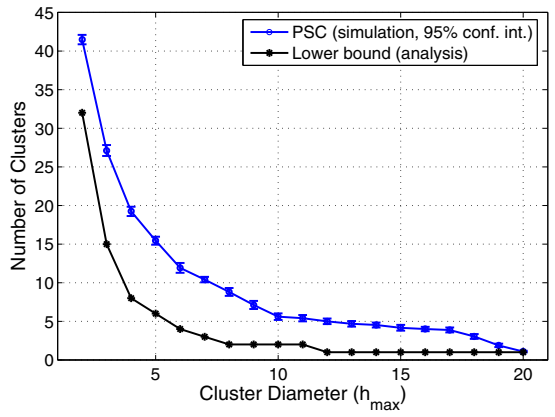

Fig. 3. Number of clusters for various $h_{\max }$

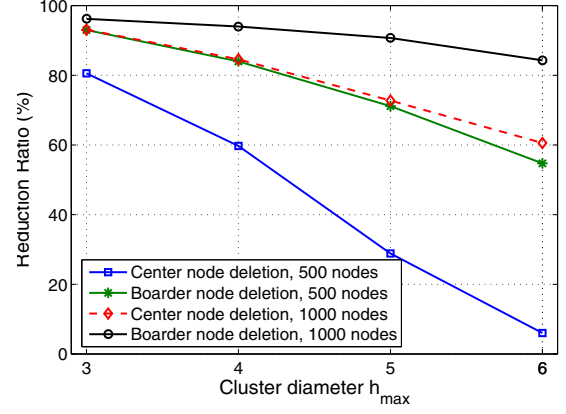

Fig. 4. Computation cost reduction using $A^{\prime \prime}$. routers is $60 \mathrm{~m}$. The cluster diameter bound is $h_{\max }=4$. After applying PSC to this network, the mesh routers are divided into 20 clusters, as illustrated in the lower tier of Fig. 2. The upper tier in Fig. 2 is constructed by GEA.

In Fig. 3, we plot the number of clusters formed by PSC for increasing cluster diameter bound $h_{\max }$, where the same setting as in Fig. 2 is used. For each $h_{\max }$ value, we randomly generate 25 different topologies for the mesh network. Each point on the PSC curve is the average of the 25 topologies, with 95\% confidence intervals plotted as error bars. The confidence intervals are generally negligible, implying that the PSC performance is quite stable. As expected, the number of clusters quickly decreases for increased $h_{\max }$, and converges to one when $h_{\max }=20$. We also plot the lower bound as given in Theorem 1. The lower bounding curve lies below the PSC curve and is reasonably tight for practical systems.

Finally we examine the computational cost reduction as given in Theorem 2 and Corollaries 2.1 and 2.2. Let $G^{\prime \prime} \subseteq G^{\prime}$ be the subgraph corresponding to $\mathbf{A}^{\prime \prime}$. We define the computation reduction ratio as $\left[\left|V\left(G^{\prime}\right)\right|-\left|V\left(G^{\prime \prime}\right)\right|\right] /\left|V\left(G^{\prime}\right)\right| \times$ $100(\%)$. A larger ratio implies multiplication of smaller matrices and larger computation reduction. Fig. 4 shows the reduction ratios by deleting a node from a 500-node network and a 1,000-node network. It can be seen that deleting a border nodes results in smaller $\mathbf{A}^{\prime \prime}$ than deleting a central node, and more reductions can be achieved for larger networks.

\section{B. Topology Optimization Results}

When the clusters are formed, location and degree information of cluster heads are used as input to GEA for optimizing the top tier topology. We assume $\Gamma_{t h}=0.9$, $I / I_{0}=0.8$, and $\lambda=1,550 \mathrm{~nm}$. The link reliability $\Gamma_{i j}$ and weight $\omega_{i j}$ are determined by distance and weather condition using the FSO channel model. The distances are computed from cluster head locations. For weather condition, the index of refraction structure parameter $C_{n}^{2}$ is randomly generated in $\left[10^{-16}, 10^{-14}\right] \mathrm{m}^{-2 / 3}$. The typical weather condition is $C_{n}^{2}=10^{-15} \mathrm{~m}^{-2 / 3}$.

We first examined a small network with the same setting as that in Fig. 2 (but with a different random topology). With cluster diameter bound $h_{\max }=3$, the mesh routers are divided into 26 clusters. The traffic demand requires that $m=39$ FSO edges should be added in the top tier, and the total degree is

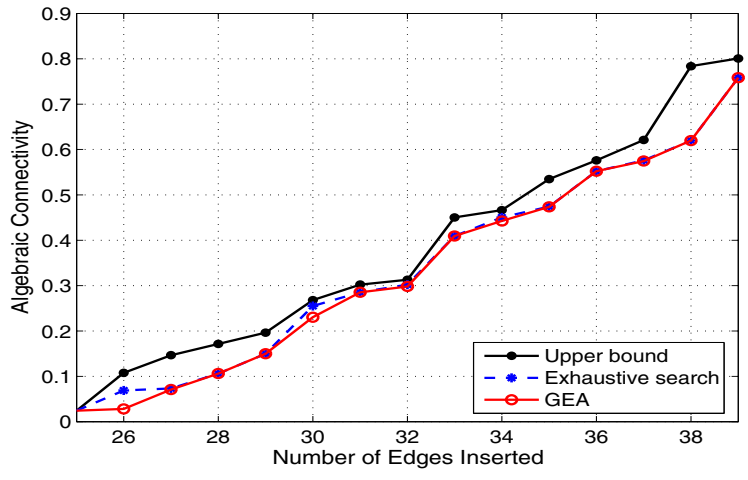

Fig. 5. Algebraic connectivity vs. number of edges for the small network.

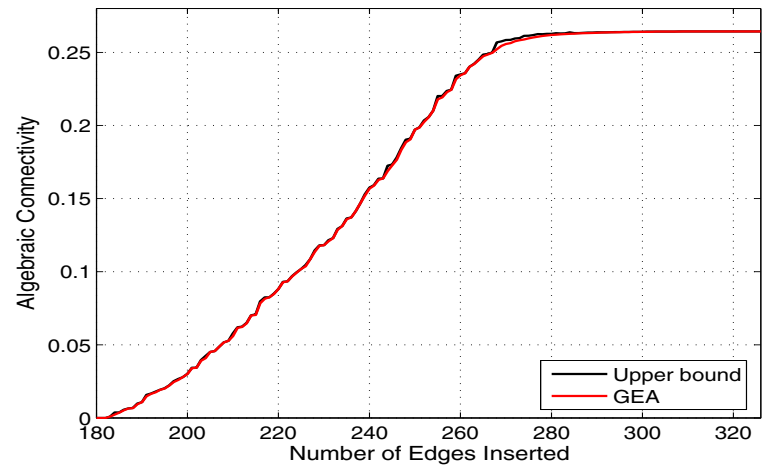

Fig. 6. Algebraic connectivity vs. number of edges for the large network.

$\sum_{i=1}^{26} K_{i}=2 \cdot m=78$. We first execute the enhanced Prim's algorithm to build a degree bounded minimum spanning tree using 25 edges (solid lines). GEA then iteratively inserts 14 edges to the tree graph.

In Fig. 5, we plot the resulting algebraic connectivity after each edge is appended. For comparison, we also plot the upper bound as given in Theorem 3 and the global optimal values found by an exhaustive search, which is possible for this small network. We find that the upper bound provides a very good approximation for the global optimum. The GEA curve overlaps with the global optimal curve for most of the iterations (except for three points). When GEA terminates, the upper bounding algebraic connectivity is 0.8006 and the GEA value is 0.7583 , which is identical to the global optimum.

We next design a large-sized network deployed in a 6,000 $\times$ $6,000 \mathrm{~m}^{2}$ area. The PSC algorithm forms 184 clusters, and 326 
edges are to be inserted. Building the spanning tree uses 183 edges, and the remaining 143 edges are iteratively appended by GEA. In Fig. 6, we plot the trace of algebraic connectivity as edges are inserted. Due to the large size, exhaustive search is not feasible. We plot the upper bound as given in (27). We find the GEA curve overlaps with the upper bound curve for most of the iterations. When GEA terminates, both the upper bound and the GEA values are 0.2643. Clearly, not only the upper bound is highly tight, the greedy heuristic GEA algorithm also achieves the global optimum for this network.

\section{RELATED WORK}

This paper is closely related to the class of network planning work. Network planning problems usually belong to the class of combinatorial optimization problems, which are NP-hard, and metaheuristics, e.g., simulated annealing [19] or genetic algorithms [20] are used to provide sub-optimal solutions. The main limitations of these approaches are the lack of performance guarantees and relatively high complexity.

This work is also related to FSO research. See excellent surveys in [10], [21] and references therein. Major FSO research has focused on the PHY so far, such as hardware architecture [21] and optical channel modeling [11]. Recently, there have been several work on the design [22] and (re)configuration [23], [24] of FSO networks. As in network planning, ILPs are usually formulated and various heuristic algorithms are proposed to provide sub-optimal solutions.

WMNs are known to suffer from capacity and fairness problems, especially when size grows [1]. A hierarchical network architecture represents a solution to the scalability problem, as observed in wireless sensor networks [4]. In [12], the authors propose a recursive dominating set (DS) selection algorithm under delay and bandwidth constraints. Genetic algorithms is used in [20] to improve the capacity performance with minimum number of hybrid FSO/RF gateways.

Algebraic connectivity is a useful tool from spectral graph theory [14], [17]. It has been used in several papers as measure of connectivity [7], [8]. In an interesting work [8], Ghosh and Boyd present an SDP formulation as well as a greedy perturbation heuristic for adding edges into existing graph, with the objective of maximize its algebraic connectivity. In [7], the authors propose a multi-level algorithm for finding the the best locations for a given set of relays, for enhancing the connectivity of wireless sensor networks. A standard SDP problem is formulated and solved in each level.

\section{CONCLUSION}

We studied the problem of design and optimization of a tiered wireless access network. The lower tier mesh network is first partitioned into clusters, and then the topology of the upper tier FSO network is optimized. The objective is to maximize connectivity (and thus robustness) while meeting traffic demand and delay requirements. We presented a PSC algorithm for the clustering problem and a GEA algorithm for the topology optimization problem and derived bounds on their performance. Our simulation studies show the algorithms are highly effective for the design and optimization of the tiered wireless access network.

\section{ACKNOWLEDGMENTS}

This work is supported in part by the National Science Foundation under Grants ECCS-0802113 and CNS-0855251, and through the Wireless Internet Center for Advanced Technology at Auburn University (NSF Grant IIP-0738088).

\section{REFERENCES}

[1] I. F. Akyildiz, X. Wang, and W. Wang, "Wireless mesh networks: A survey," Computer Netw., vol. 47, no. 4, pp.445-487, Mar. 2005.

[2] J. Jun and M. Sichitiu, "The nominal capacity of wireless mesh networks," IEEE Wireless Commun., vol. 10, no. 5, pp.8-14, Oct. 2003.

[3] J. Shi, O. Gurewitz, V. Mancuso, J. Camp, and E. Knightly, "Measurement and modeling of the origins of starvation in congestion controlled mesh networks," in Proc. IEEE INFOCOM'08, Phoenix, AZ, Apr. 2008, pp.1633-1641.

[4] A. A. Abbasi and M. Younis, "A survey on clustering algorithms for wireless sensor networks," Computer Commun., vol. 30, no. 14/15, pp.2826-2841, Oct. 2007.

[5] B. Liu, Z. Liu, and D. Towsley, "On the capacity of hybrid wireless networks," in Proc. IEEE INFOCOM'03, San Francisco, CA, Mar. 2003, pp.1543-1552.

[6] M. R. Garey and D. S. Johnson, Computers and Intractability: A Guide to the Theory of NP-Completeness. New York, NY: W.H. Freeman \& Co., 1990.

[7] A. S. Ibrahim, K. G. Seddik, and K. R. Liu, "Improving connectivity via relays deployment in wireless sensor networks," in Proc. IEEE GLOBECOM'07, Washington, DC, Nov. 2007, pp.1159-1163.

[8] A. Ghosh and S. Boyd, "Growing well-connected graphs," in Proc. IEEE CDC'06, San Diego, CA, Dec. 2006, pp.6605-6611.

[9] E. Korevaar, I. I. Kim, and B. McArthur, "Atmospheric propagation characteristics of highest importance to commercial free space optics," in Proc. Int. Congress Math., Bulgaria, Cyprus, Sept. 2003, pp.1-12.

[10] D. J. Heatley, D. R. Wisely, I. Neild, and P. Cochrane, "Optical wireless: The story so far," IEEE Commun., vol. 36, no. 12, pp.72-82, Dec. 1998.

[11] X. Zhu and J. M. Kahn, "Free-space optical communication through atmospheric turbulence channels," IEEE Trans. Commun., vol. 50, no. 8 , pp.1293-1300, Mar. 2003.

[12] B. Aoun, R. Boutaba, Y. Iraqi, and G. Kenward, "Gateway placement optimization in wireless mesh networks with QoS constraints," IEEE J. Sel. Areas Commun., vol. 24, no. 11, pp.2127-2136, Nov. 2006.

[13] J. L. Gross and J. Yellen, Graph Theory and Its Applications. New York, NY: CRC Press, 1999.

[14] F. R. K. Chung, Spectral Graph Theory. Providence, RI: American Mathematical Society, 1997.

[15] M. Fiedler, "Algebraic connectivity of graphs," Czechoslovak Mathematical Journal, vol. 23, no. 2, pp.298-305, 1973.

[16] B. Mohar, "The Laplacian spectrum of graphs," in Graph Theory, Combinatorics, and Applications, Y. Alavi, G. Chartrand, O. Oellermann, and A. Schwenk, Eds. Hoboken, NJ: Wiley, 1991, pp.871-898.

[17] S. Boyd, "Convex optimization of graph Laplacian eigenvalues," in Proc. Int. Congress Math., Madrid, Spain, Aug. 2006, pp.1311-1319.

[18] R. Jothi and B. Raghavachari, "Degree-bounded minimum spanning trees," Discrete Appl. Math., vol. 157, no. 5, pp.960-970, Mar. 2009.

[19] C. Ersoy and S. Panwar, "Topological design of interconnected LANMAN networks," IEEE J. Sel. Areas Commun., vol. 11, no. 8, pp.11721182, Oct. 1993.

[20] M. N. Smadi, S. C. Ghosh, A. A. Farid, T. D. Todd, and S. Hranilovic, "Free-space optical gateway placement in hybrid wireless mesh networks," IEEE/OSA J. Lightw. Technol., to appear.

[21] V. W. Chan, "Free-space optical communications," IEEE/OSA J. Lightw. Technol., vol. 24, no. 12, pp.4750-4762, Dec. 2006.

[22] P. C. Gurumohan and J. Hui, "Topology design for free space optical networks," Proc. IEEE ICCCN'03, Dallas, TX, Oct. 2003, pp.576-579.

[23] F. Liu, U. Vishkin, and S. Milner, "Bootstrapping free-space optical networks," IEEE J. Sel. Areas Commun., vol. 24, no. 12, pp.13-22, Dec. 2006.

[24] A. Desai and S. Milner, "Autonomous reconfiguration in free-space optical sensor networks," IEEE J. Sel. Areas Commun., vol. 23, no. 8, pp.1556-1563, Aug. 2005. 